PUBLICATIONS DE L'INSTITUT MATHÉMATIQUE

Nouvelle série, tome 76(90) (2004), 81-88

\title{
AN ASYMPTOTIC FORMULA FOR A SUM INVOLVING ZEROS OF THE RIEMANN ZETA-FUNCTION
}

\author{
Yuichi Kamiya and Masatoshi Suzuki
}

\begin{abstract}
E. Landau gave an interesting asymptotic formula for a sum involving zeros of the Riemann zeta-function. We give an asymptotic formula which can be regarded as a smoothed version of Landau's formula.
\end{abstract}

\section{Introduction}

Let $\zeta(s)$ be the Riemann zeta-function. It is important to study non-trivial zeros $\rho=\beta+i \gamma$ of $\zeta(s)$. Weil's explicit formula is one of useful formulas for the study of $\rho$. Roughly speaking, it connects certain sums involving $\rho$ with sums involving prime numbers in terms of test functions and those Mellin transforms. We can refer to Lang [6] or Patterson [7] for the details of Weil's explicit formula.

In this paper, as an application of Weil's explicit formula with a certain test function, we shall study the asymptotic behaviour of a quantity involving $\rho$, that is,

$$
\sum_{\rho} e^{u \rho^{2}-v \rho}
$$

Some suitable choice of the test function enables us to get asymptotic formulas for (1.1).

TheOREM 1.1. (i) For $v=u$ or $v=0$ we have

$$
\sum_{\rho} e^{u \rho^{2}-v \rho}=\frac{1}{\sqrt{16 \pi u}} \log \frac{1}{u}-\frac{\log \left(16 \pi^{2}\right)+\mathcal{C}}{\sqrt{16 \pi u}}+O(1), \quad u \rightarrow+0,
$$

where $\mathcal{C}$ is the Euler constant, and the sum $\sum_{\rho}$ runs over all non-trivial zeros $\rho$ counting with multiplicity.

(ii) For any integer $m \geqslant 2$ we have

$$
\sum_{\rho} e^{u \rho^{2}+(\log m) \rho}=-\frac{\Lambda(m)}{\sqrt{4 \pi u}}+O(1), \quad u \rightarrow+0,
$$

2000 Mathematics Subject Classification: 11M36.

Key words and phrases: Riemann zeta-function, non-trivial zeros, asymptotic formula. 
where $\Lambda(m)=\log p$ if $m$ is a power of a prime $p$ and $\Lambda(m)=0$ otherwise. The implied constant depends on $m$.

(ii) $^{\prime}$ Let $K$ be a closed interval contained in $(-\infty, 0)-\bigcup_{m}\{-\log m\}$, where $m$ is a power of a prime. Then we have

$$
\sum_{\rho} e^{u \rho^{2}-v \rho}=O(1), \quad u \rightarrow+0,
$$

uniformly for $v$ in $K$.

(iii) For any integer $m \geqslant 2$ we have

$$
\sum_{\rho} e^{u \rho^{2}-(\log m) \rho}=-\frac{\Lambda(m)}{m \sqrt{4 \pi u}}+O(1), \quad u \rightarrow+0 .
$$

The implied constant depends on $m$.

(iii)' Let $K$ be a closed interval contained in $(0, \infty)-\cup_{m}\{\log m\}$, where $m$ is a power of a prime. Then we have

$$
\sum_{\rho} e^{u \rho^{2}-v \rho}=O(1), \quad u \rightarrow+0
$$

uniformly $v$ in $K$.

We can see asymptotic behaviours different from each other for the quantity (1.1) and the difference depends on the choice of $v$. The first and second terms on the right-hand side of the asymptotic formula in (i) come from the logarithmic derivative of the gamma factor appeared in the functional equation of $\zeta(s)$. On the other hand, the first terms on the right-hand sides of the asymptotic formulas in (ii) and (iii) come from the logarithmic derivative of $\zeta(s)$.

The asymptotic formula in (ii) is related to the results of Landau [5], Gonek [3] [4], and Fujii [2]. Landau [5] proved that, for fixed $x>1$,

$$
\sum_{0<\gamma \leqslant T} x^{\rho}=-\frac{T}{2 \pi} \Lambda(x)+O(\log T)
$$

holds. Gonek [3] [4] gave uniform versions of Landau's result, and Fujii [2] gave a refined formula for it under the Riemann Hypothesis. The asymptotic formula in (ii) may be regarded as a smoothed version of Landau's with the measure given by the Gaussian function.

The asymptotic formula in (i) may be regarded as a smoothed version of the asymptotic formula for $N(T)$, number of non-trivial zeros $\rho$ with $0<\gamma<T$. To see this, let us consider the case $v=u$ in (i) under the Riemann Hypothesis. Then the asypmtotic formula in (i) is

$$
\sum_{\gamma} e^{-u\left(1 / 4+\gamma^{2}\right)}=\frac{1}{\sqrt{16 \pi u}} \log \frac{1}{u}-\frac{\log \left(16 \pi^{2}\right)+\mathcal{C}}{\sqrt{16 \pi u}}+O(1) .
$$


The sum on the left-hand side is written as an integral form, and, by integration by parts, it follows that

$$
-\int_{0}^{\infty} N(T) d\left(e^{-u T^{2}}\right)=\frac{1}{2 \sqrt{16 \pi u}} \log \frac{1}{u}-\frac{\log \left(16 \pi^{2}\right)+\mathcal{C}}{2 \sqrt{16 \pi u}}+O(1) .
$$

The authors would like to thank Professor Kohji Matsumoto for reading the manuscript. They would like to thank the referee for kind comments.

\section{An explicit formula for a sum involving zeros of the Riemann zeta-function}

In this section we give an explicit formula, which is a variant of Weil's explicit formula.

Lemma 2.1. For any positive $u$ and any real $v$ we have

$$
\begin{aligned}
\sum_{\rho} e^{u \rho^{2}-v \rho}= & e^{u-v}-\frac{\log \pi}{\sqrt{4 \pi u}} e^{-v^{2} / 4 u}+1 \\
& -\frac{1}{\sqrt{4 \pi u}} \sum_{n=2}^{\infty} \Lambda(n) e^{-(v+\log n)^{2} / 4 u}-\frac{1}{\sqrt{4 \pi u}} \sum_{n=2}^{\infty} \frac{\Lambda(n)}{n} e^{-(v-\log n)^{2} / 4 u} \\
& +\frac{e^{u / 4-v / 2}}{2 \pi} \int_{-\infty}^{\infty} \log \left|\frac{1}{4}+i \frac{t}{2}\right| \cdot e^{-u t^{2}+i t(u-v)} d t-\left(E * G_{u}\right)(v),
\end{aligned}
$$

where the functions $E$ and $G_{u}$ are defined by

$$
E(x)=\left(\frac{1}{e^{2|x|}-1}-\frac{1}{2|x|}+1\right) e^{-|x| / 2-x / 2}, \quad G_{u}(x)=\frac{1}{\sqrt{4 \pi u}} e^{-x^{2} / 4 u},
$$

and $E * G_{u}$ means the convolution of $E$ and $G_{u}$, that is,

$$
\left(E * G_{u}\right)(v)=\int_{-\infty}^{\infty} E(x) G_{u}(v-x) d x .
$$

Proof. Since

$$
\int_{0}^{\infty} \frac{1}{\sqrt{4 \pi u}} e^{-(v+\log x)^{2} / 4 u} x^{s} \frac{d x}{x}=e^{u s^{2}-v s}
$$

we have

$$
\frac{1}{2 \pi i} \int_{1+\delta-i \infty}^{1+\delta+i \infty} \frac{\zeta^{\prime}}{\zeta}(s) e^{u s^{2}-v s} d s=-\sum_{n=2}^{\infty} \Lambda(n) \frac{1}{\sqrt{4 \pi u}} e^{-(v+\log n)^{2} / 4 u} .
$$

We also have

$$
\frac{1}{2 \pi i} \int_{1+\delta-i \infty}^{1+\delta+i \infty} \frac{\zeta^{\prime}}{\zeta}(s) e^{u s^{2}-v s} d s=\frac{1}{2 \pi i} \int_{-\delta-i \infty}^{-\delta+i \infty} \frac{\zeta^{\prime}}{\zeta}(s) e^{u s^{2}-v s} d s+\sum_{\rho} e^{u \rho^{2}-v \rho}-e^{u-v} .
$$


The first term on the right-hand side can be expressed in the following form by the functional equation of $\zeta(s)$ :

$$
\begin{aligned}
\frac{1}{2 \pi i} & \int_{-\delta-i \infty}^{-\delta+i \infty}\left(-\frac{\zeta^{\prime}}{\zeta}(1-s)+\log \pi-\frac{1}{2} \frac{\Gamma^{\prime}}{\Gamma}\left(\frac{s}{2}\right)-\frac{1}{2} \frac{\Gamma^{\prime}}{\Gamma}\left(\frac{1-s}{2}\right)\right) e^{u s^{2}-v s} d s \\
= & \frac{1}{\sqrt{4 \pi u}} \sum_{n=2}^{\infty} \frac{\Lambda(n)}{n} e^{-(v-\log n)^{2} / 4 u}+\frac{\log \pi}{\sqrt{4 \pi u}} e^{-v^{2} / 4 u}-1 \\
& -\frac{1}{4 \pi} \int_{-\infty}^{\infty}\left(\frac{\Gamma^{\prime}}{\Gamma}\left(\frac{1}{4}+i \frac{t}{2}\right)+\frac{\Gamma^{\prime}}{\Gamma}\left(\frac{1}{4}-i \frac{t}{2}\right)\right) e^{u(1 / 2+i t)^{2}-v(1 / 2+i t)} d t .
\end{aligned}
$$

Hence we have

$$
\begin{aligned}
\sum_{\rho} e^{u \rho^{2}-v \rho}= & e^{u-v}-\frac{\log \pi}{\sqrt{4 \pi u}} e^{-v^{2} / 4 u}+1 \\
& -\frac{1}{\sqrt{4 \pi u}} \sum_{n=2}^{\infty} \Lambda(n) e^{-(v+\log n)^{2} / 4 u}-\frac{1}{\sqrt{4 \pi u}} \sum_{n=2}^{\infty} \frac{\Lambda(n)}{n} e^{-(v-\log n)^{2} / 4 u} \\
& +\frac{e^{u / 4-v / 2}}{4 \pi} \int_{-\infty}^{\infty}\left(\frac{\Gamma^{\prime}}{\Gamma}\left(\frac{1}{4}+i \frac{t}{2}\right)+\frac{\Gamma^{\prime}}{\Gamma}\left(\frac{1}{4}-i \frac{t}{2}\right)\right) e^{-u t^{2}+i t(u-v)} d t
\end{aligned}
$$

This formula is a special case of Weil's explicit formula (with the test function $\left.\frac{1}{\sqrt{4 \pi u}} e^{-(v+\log x)^{2} / 4 u}\right)$, but we supply a proof to make the paper self-contained.

Let us denote the last term on the right-hand side by $H$. By the expression (see, for example, $[\mathbf{1}$, p. 28, l. 16])

$$
\frac{\Gamma^{\prime}}{\Gamma}(z)=\log z-\int_{0}^{\infty}\left(\frac{1}{e^{x}-1}-\frac{1}{x}+1\right) e^{-z x} d x, \quad \operatorname{Re} z>0,
$$

we have

$$
\begin{aligned}
H= & \frac{e^{u / 4-v / 2}}{4 \pi} \int_{-\infty}^{\infty}\left(\log \left(\frac{1}{4}+i \frac{t}{2}\right)+\log \left(\frac{1}{4}-i \frac{t}{2}\right)\right) e^{-u t^{2}+i t(u-v)} d t \\
& -\frac{e^{u / 4-v / 2}}{4 \pi} \int_{0}^{\infty}\left(\frac{1}{e^{x}-1}-\frac{1}{x}+1\right) e^{-x / 4} \sqrt{\frac{\pi}{u}}\left(e^{-\frac{(u-v-x / 2)^{2}}{4 u}}+e^{-\frac{(u-v+x / 2)^{2}}{4 u}}\right) d x \\
= & \frac{e^{u / 4-v / 2}}{2 \pi} \int_{-\infty}^{\infty} \log \left|\frac{1}{4}+i \frac{t}{2}\right| \cdot e^{-u t^{2}+i t(u-v)} d t \\
& -\int_{-\infty}^{\infty}\left(\frac{1}{e^{2|x|}-1}-\frac{1}{2|x|}+1\right) e^{-|x| / 2-x / 2} \frac{1}{\sqrt{4 \pi u}} e^{-(v-x)^{2} / 4 u} d x
\end{aligned}
$$

Hence we obtain the lemma.

\section{Proof of Theorem}

To obtain the estimates in the theorem we consider separately each term on the right-hand side of Lemma 2.1.

Lemma 3.1. We have $0 \leqslant\left(E * G_{u}\right)(v) \leqslant 1$. 
Proof. It is easy to verify that $0 \leqslant E(x) \leqslant 1$. Hence

$$
0 \leqslant\left(E * G_{u}\right)(v) \leqslant \int_{-\infty}^{\infty} G_{u}(x) d x=1 .
$$

Here, we remark on the convolution $\left(E * G_{u}\right)(v)$. The assertion of Lemma 2.1 is enough for the proof of the theorem, but we can obtain a more precise behaviour of the convolution. It is not hard to verify that the function $E$ has the property $|E(v-x)-E(v)| \leqslant C|x|$, where $C$ is a positive absolute constant. Hence we have

$$
\begin{aligned}
\left|\left(E * G_{u}\right)(v)-E(v)\right| & \leqslant \int_{-\infty}^{\infty}|E(v-x)-E(v)| G_{u}(x) d x \\
& \leqslant C \int_{-\infty}^{\infty}|x| G_{u}(x) d x=C \sqrt{\frac{4 u}{\pi}}
\end{aligned}
$$

that is, $\left(E * G_{u}\right)(v)=E(v)+O(\sqrt{u})$.

The next lemma is the key for the proof of the theorem.

Lemma 3.2. For $0<u<1$ we have

$$
\begin{aligned}
\int_{-\infty}^{\infty} \log \mid \frac{1}{4}+ & i \frac{t}{2} \mid \cdot e^{-u t^{2}+i t(u-v)} d t \\
& = \begin{cases}O\left(\frac{1}{|u-v|^{2}}\right) & \text { if } v \neq u \text { and } v \neq 0, \\
\sqrt{\frac{\pi}{4 u}} \log \frac{1}{u}-\sqrt{\frac{\pi}{4 u}}(4 \log 2+\mathcal{C})+O(1), & \text { if } v=u \text { or } v=0,\end{cases}
\end{aligned}
$$

where the implied constants are absolute.

Proof. Firstly, we consider the case $v \neq u$ and $v \neq 0$. We have

$$
\begin{aligned}
& \int_{-\infty}^{\infty} \log \left|\frac{1}{4}+i \frac{t}{2}\right| \cdot e^{-u t^{2}+i t(u-v)} d t \\
& =\int_{0}^{\infty} \log \left(\frac{1}{16}+\frac{t^{2}}{4 u}\right) \cdot e^{-t^{2}} \cos \left(\frac{t(u-v)}{\sqrt{u}}\right) \frac{d t}{\sqrt{u}} \\
& =-\frac{1}{u-v} \int_{0}^{\infty} \frac{d}{d t}\left(\log \left(\frac{1}{16}+\frac{t^{2}}{4 u}\right) \cdot e^{-t^{2}}\right) \sin \left(\frac{t(u-v)}{\sqrt{u}}\right) d t \\
& =-\frac{\sqrt{u}}{(u-v)^{2}} \int_{0}^{\infty} \frac{d^{2}}{d t^{2}}\left(\log \left(\frac{1}{16}+\frac{t^{2}}{4 u}\right) \cdot e^{-t^{2}}\right) \cos \left(\frac{t(u-v)}{\sqrt{u}}\right) d t \\
& =-\frac{\sqrt{u}}{(u-v)^{2}}\left\{\int_{0}^{\infty} \frac{2\left(-t^{2}+u / 4\right)}{\left(t^{2}+u / 4\right)^{2}} e^{-t^{2}} \cos \left(\frac{t(u-v)}{\sqrt{u}}\right) d t\right. \\
& \quad-\int_{0}^{\infty} \frac{8 t^{2}}{t^{2}+\frac{u}{4}} e^{-t^{2}} \cos \left(\frac{t(u-v)}{\sqrt{u}}\right) d t \\
& \left.\quad+\int_{0}^{\infty} \log \left(\frac{1}{16}+\frac{t^{2}}{4 u}\right) \cdot e^{-t^{2}}\left(4 t^{2}-2\right) \cos \left(\frac{t(u-v)}{\sqrt{u}}\right) d t\right\} \\
& =-\frac{\sqrt{u}}{(u-v)^{2}}\left\{I_{1}+I_{2}+I_{3}\right\},
\end{aligned}
$$

say. As for $I_{1}$ and $I_{2}$ we easily have 


$$
\begin{aligned}
& \left|I_{1}\right| \leqslant 2 \int_{0}^{\infty} \frac{1}{t^{2}+u / 4} e^{-t^{2}} d t \leqslant \frac{8}{u} \int_{0}^{\sqrt{u}} d t+2 \int_{\sqrt{u}}^{\infty} \frac{1}{t^{2}} d t=10 \frac{1}{\sqrt{u}}, \\
& \left|I_{2}\right| \leqslant 8 \int_{0}^{\infty} e^{-t^{2}} d t=4 \sqrt{\pi} .
\end{aligned}
$$

As for $I_{3}$ we have

$$
\begin{aligned}
\left|I_{3}\right| \leqslant & \int_{0}^{\infty}\left|\log \left(\frac{1}{16}+\frac{t^{2}}{4 u}\right)\right| e^{-t^{2}}\left(4 t^{2}+2\right) d t \\
\leqslant & \log \frac{1}{u} \cdot \int_{0}^{\infty} e^{-t^{2}}\left(4 t^{2}+2\right) d t+\int_{0}^{2}\left|\log \left(\frac{u}{16}+\frac{t^{2}}{4}\right)\right| e^{-t^{2}}\left(4 t^{2}+2\right) d t \\
& +\int_{2}^{\infty}\left|\log \left(\frac{u}{16}+\frac{t^{2}}{4}\right)\right| e^{-t^{2}}\left(4 t^{2}+2\right) d t \\
\ll & \log \frac{1}{u}+\int_{2}^{\infty} \log t \cdot e^{-t^{2}}\left(4 t^{2}+2\right) d t \ll \log \frac{1}{u} .
\end{aligned}
$$

Substituting (3.2), (3.3), and (3.4) into (3.1), we obtain the first estimate of this lemma.

Next, we consider the case $v=u$. We have

$$
\begin{aligned}
& \int_{-\infty}^{\infty} \log \left|\frac{1}{4}+i \frac{t}{2}\right| \cdot e^{-u t^{2}} d t \\
& =\int_{0}^{\infty} \log \left(\frac{1}{16}+\frac{t^{2}}{4 u}\right) \cdot e^{-t^{2}} \frac{d t}{\sqrt{u}} \\
& =\frac{\log \frac{1}{u}}{\sqrt{u}} \int_{0}^{\infty} e^{-t^{2}} d t+\frac{1}{\sqrt{u}} \int_{0}^{\infty} \log \left(\frac{u}{16}+\frac{t^{2}}{4}\right) \cdot e^{-t^{2}} d t \\
& =\frac{\sqrt{\pi}}{2} \frac{\log \frac{1}{u}}{\sqrt{u}}+\frac{1}{\sqrt{u}} \int_{0}^{\infty} \log \frac{t^{2}}{4} \cdot e^{-t^{2}} d t+\frac{1}{\sqrt{u}} \int_{0}^{\infty} \log \left(1+\frac{u}{4 t^{2}}\right) \cdot e^{-t^{2}} d t \\
& =\frac{\sqrt{\pi}}{2} \frac{\log \frac{1}{u}}{\sqrt{u}}+\frac{1}{\sqrt{u}} J_{1}+\frac{1}{\sqrt{u}} J_{2},
\end{aligned}
$$

say. As for $J_{2}$ we have

$$
\begin{aligned}
J_{2} & =\int_{0}^{\sqrt{u}} \log \left(1+\frac{u}{4 t^{2}}\right) \cdot e^{-t^{2}} d t+\int_{\sqrt{u}}^{\infty} \log \left(1+\frac{u}{4 t^{2}}\right) \cdot e^{-t^{2}} d t \\
& \leqslant \int_{0}^{\sqrt{u}} \log \left(1+\frac{u}{4 t^{2}}\right) d t+\frac{u}{4} \int_{\sqrt{u}}^{\infty} \frac{1}{t^{2}} e^{-t^{2}} d t \\
& =\sqrt{u} \log \frac{5}{4}+\int_{0}^{\sqrt{u}} \frac{2 u}{4 t^{2}+u} d t+\frac{u}{4} \int_{\sqrt{u}}^{\infty} \frac{1}{t^{2}} e^{-t^{2}} d t \\
& \leqslant \sqrt{u} \log \frac{5}{4}+2 \sqrt{u}+\frac{u}{4} \int_{\sqrt{u}}^{\infty} \frac{1}{t^{2}} d t \ll \sqrt{u} .
\end{aligned}
$$


For $J_{1}$ we have

$$
\begin{aligned}
J_{1} & =\int_{0}^{\infty} \log t \cdot e^{-t} \frac{d t}{2 \sqrt{t}}-\log 4 \cdot \int_{0}^{\infty} e^{-t^{2}} d t \\
& =\frac{1}{2} \Gamma^{\prime}\left(\frac{1}{2}\right)-\sqrt{\pi} \log 2=-\frac{\sqrt{\pi}}{2}(4 \log 2+\mathcal{C})
\end{aligned}
$$

Substituting (3.6) and (3.7) into (3.5), we obtain the second asymptotic formula in this lemma in the case $v=u$.

Finally, we consider the case $v=0$. We have

$$
\begin{aligned}
& \int_{-\infty}^{\infty} \log \left|\frac{1}{4}+i \frac{t}{2}\right| \cdot e^{-u t^{2}+i t u} d t \\
& =\int_{0}^{\infty} \log \left(\frac{1}{16}+\frac{t^{2}}{4 u}\right) \cdot e^{-t^{2}} \cos (\sqrt{u} t) \frac{d t}{\sqrt{u}} \\
& =\frac{\log \frac{1}{u}}{\sqrt{u}} \int_{0}^{\infty} e^{-t^{2}} \cos (\sqrt{u} t) d t+\frac{1}{\sqrt{u}} \int_{0}^{\infty} \log \frac{t^{2}}{4} \cdot e^{-t^{2}} \cos (\sqrt{u} t) d t \\
& \quad+\frac{1}{\sqrt{u}} \int_{0}^{\infty} \log \left(1+\frac{u}{4 t^{2}}\right) \cdot e^{-t^{2}} \cos (\sqrt{u} t) d t \\
& =\frac{\log \frac{1}{u}}{\sqrt{u}} K_{1}+\frac{1}{\sqrt{u}} K_{2}+\frac{1}{\sqrt{u}} K_{3}
\end{aligned}
$$

say. As for $K_{3}$ we have

$$
\left|K_{3}\right| \leqslant J_{2} \ll \sqrt{u}
$$

For $K_{1}$ and $K_{2}$ we use

$$
\cos (\sqrt{u} t)=1+O\left(u t^{2}\right)
$$

From (3.10) it follows that

$$
\begin{aligned}
K_{1} & =\int_{0}^{\infty} e^{-t^{2}} d t+O\left(u \int_{0}^{\infty} e^{-t^{2}} t^{2} d t\right)=\frac{\sqrt{\pi}}{2}+O(u), \\
K_{2} & =\int_{0}^{\infty} \log \frac{t^{2}}{4} \cdot e^{-t^{2}} d t+O\left(u \int_{0}^{\infty} \log \frac{t^{2}}{4} \cdot e^{-t^{2}} t^{2} d t\right) \\
& =-\frac{\sqrt{\pi}}{2}(4 \log 2+\mathcal{C})+O(u) .
\end{aligned}
$$

Substituting (3.9), (3.11), and (3.12) into (3.8), we obtain the second asymptotic formula in this lemma in the case $v=0$.

To obtain the theorem we now consider the asymptotic behaviour of the quantity

$$
-\frac{1}{\sqrt{4 \pi u}} \sum_{n=2}^{\infty} \Lambda(n) e^{-(v+\log n)^{2} / 4 u}-\frac{1}{\sqrt{4 \pi u}} \sum_{n=2}^{\infty} \frac{\Lambda(n)}{n} e^{-(v-\log n)^{2} / 4 u}
$$


in Lemma 2.1. The behaviour of this quantity depends on the choice of $v$. For the case $v=0$ and $0<u<1$

$$
e^{-(\log n)^{2} / 4 u}=e^{-(\log n)^{2} / 8 u} e^{-(\log n)^{2} / 8 u} \leqslant e^{-(\log n)^{2} / 8} e^{-(\log 2)^{2} / 8 u},
$$

and hence (3.13) is of exponential decay as $u \rightarrow+0$. For the case $v=-\log m$, $m \geqslant 2$ is an integer, and $0<u<1$ we have

$$
\begin{aligned}
e^{-\frac{1}{4 u}(-\log m+\log n)^{2}} & \leqslant e^{-\frac{1}{8 u}(-\log m+\log n)^{2}} e^{-\frac{1}{8 u}(-\log m+\log (m+1))^{2}} \\
& \leqslant e^{-\frac{1}{8}(\log n)^{2}\left(1-\frac{\log m}{\log n}\right)^{2}} e^{-\frac{1}{8 u}(-\log m+\log (m+1))^{2}}, \quad n \neq m,
\end{aligned}
$$

and

$$
e^{-\frac{1}{4 u}(-\log m-\log n)^{2}} \leqslant e^{-\frac{1}{4 u}(\log n)^{2}} \leqslant e^{-\frac{1}{8}(\log n)^{2}} e^{-\frac{1}{8 u}(\log 2)^{2}},
$$

and hence (3.13) is

$$
\begin{aligned}
= & -\frac{\Lambda(m)}{\sqrt{4 \pi u}}+O\left(\frac{e^{-\frac{1}{8 u}(\log 2)^{2}}}{\sqrt{u}} \sum_{n=2}^{\infty} \frac{\Lambda(n)}{n} e^{-\frac{1}{8}(\log n)^{2}}\right. \\
& \left.+\frac{e^{-\frac{1}{8 u}(-\log m+\log (m+1))^{2}}}{\sqrt{u}}\left(\sum_{m \neq n=2}^{m^{2}} \Lambda(n)+\sum_{n>m^{2}} \Lambda(n) e^{-\frac{1}{8}(\log n)^{2} \frac{1}{4}}\right)\right) .
\end{aligned}
$$

For other $v$ we can similarly consider the asymtotic behaviour of (3.13).

Combining the above arguments and Lemmas 2.1, 3.1, and 3.2, we obtain the assertion of the theorem.

\section{References}

[1] G. Andrews, R. Askey, R. Roy, Special Functions, Cambridge University Press, 1999.

[2] A. Fujii, On a theorem of Landau. II, Proc. Japan Acad. 66 (1990), 291-296.

[3] S. Gonek, A formula of Landau and mean values of $\zeta(s)$, in: Topics in Analytic Number Theory, University of Texas Press, 1985, pp. 92-97.

[4] S. Gonek, An explicit formula of Landau and its applications to the theory of the zeta-function, in: A tribute to Emil Grosswald: Number Theory and Related Analysis, Contemp. Math. 143, Amer. Math. Soc., Providence, RI, 1993, 395-413.

[5] E. Landau, Über die Nullstellen der $\zeta$-Funktion, Math. Ann. 71 (1911), 548-568.

[6] S. Lang, Algebraic Number Theory, 2nd ed., Springer-Verlag, 1994.

[7] S. J. Patterson, An Introduction to the Theory of the Riemann Zeta-function, Cambridge University Press, 1988.

Yuichi Kamiya

19-4 Nishinobo Daiwa-cho

Okazaki-city Aichi 444-0931

Japan

kamiya-9@m3.catvmics.ne.jp

Masatoshi Suzuki

Graduate School of Mathematics

Nagoya University

Chikusa-ku, Nagoya 464-8602

Japan

m99009t@math.nagoya-u . ac.jp
(Received 1203 2004)

(Revised 0811 2004) 